\title{
Thymoma exhibiting spontaneous regression in size, pleural effusion and serum cytokeratin fragment level: A case report
}

\author{
KENTA FURUYA ${ }^{1}$, KAZUTOSHI ISOBE ${ }^{1}$, GO SANO ${ }^{1}$, KYOHEI KABURAKI ${ }^{1}$, KYOKO GOCHO ${ }^{1}$, \\ FUMIAKI ISHIDA $^{1}$, NAOSHI KIKUCHI ${ }^{1}$, KEISHI SUGINO ${ }^{1}$, SUSUMU SAKAMOTO ${ }^{1}$, \\ YUJIRO TAKAI $^{1}$, HAJIME OTSUKA ${ }^{2}$, YOSHINOBU HATA ${ }^{2}$, AKIRA IYODA $^{2}$, \\ MEGUMI WAKAYAMA ${ }^{3}$, KAZUTOSHI SHIBUYA ${ }^{3}$ and SAKAE HOMMA $^{1}$ \\ ${ }^{1}$ Division of Respiratory Medicine, ${ }^{2}$ Division of Thoracic Surgery and ${ }^{3}$ Department of Surgical Pathology, \\ Faculty of Medicine, Toho University Omori Medical Center, Tokyo 143-8541, Japan
}

Received May 22, 2015; Accepted June 11, 2015

DOI: $10.3892 / \operatorname{mco} .2015 .583$

\begin{abstract}
A 30-year-old man was admitted to Toho University Omori Medical Center for assessment of right chest pain and fever. Chest computed tomography (CT) revealed an anterior mediastinal tumor sized $11.0 \times 6.0 \times 5.0 \mathrm{~cm}$, with right pleural effusion. The laboratory analysis revealed elevated white blood cell count $(11,000 / \mu \mathrm{l}), \mathrm{C}$-reactive protein $(4.1 \mathrm{mg} / \mathrm{dl})$ and cytokeratin fragment (CYFRA; $12.7 \mathrm{ng} / \mathrm{ml}$; normal, <2 ng/ml). The level of CYFRA in the pleural effusion was also markedly elevated $(143 \mathrm{ng} / \mathrm{ml})$. On the first day after admission (6 days after the initial CT), there was a mild regression on CT (10.0x5.5x4.4 cm; reduction rate, 26.7\%), with decrease of the pleural effusion volume. A CT-guided needle biopsy was performed, but the findings were not conclusive, as most of the tissue was necrotic. Seven days later (13 days after the initial CT), a CT revealed further regression $(9.5 \times 5.4 \times 4.2 \mathrm{~cm}$; reduction rate, $34.7 \%$ ) with disappearance of the pleural effusion. The patient was followed up on an outpatient basis. At 35 days after the initial CT, the tumor continued to shrink without treatment (8.0x3.6x3.0 cm; reduction rate, $73.8 \%)$ and the serum CYFRA level had decreased to $0.8 \mathrm{ng} / \mathrm{ml}$, although it had not returned to normal levels. At 62 days after the initial CT, the patient underwent surgical resection. The resected specimen was diagnosed as thymoma (World Health Organization type B2; Masaoka classification, stage II), with prominent degeneration and necrosis. One possible cause of the spontaneous regression may be increased internal pressure, probably associated with rapid tumor growth, leading to massive necrosis with resulting chest pain, inflammatory reaction with pleural effusion and
\end{abstract}

Correspondence to: Dr Kenta Furuya and Dr Kazutoshi Isobe, Division of Respiratory Medicine, Faculty of Medicine, Toho University Omori Medical Center, 6-11-1 Omori-Nishi, Ota-ku, Tokyo 143-8541, Japan

E-mail: emmanuelpetit1717@yahoo.co.jp

E-mail: kazutoshiisobe@aol.com

Key words: thymoma, spontaneous regression, necrosis, cytokeratin fragment, pleural effusion subsequent tumor regression. The serum CYFRA level may be a useful marker for the evaluation of the clinical course of thymoma with extensive necrosis.

\section{Introduction}

Thymoma is derived from thymic epithelial cells and is one of the most common neoplasms in the anterior mediastinum (1). The overall incidence of thymoma is 0.15 cases per 100,000 individuals and the majority of the patients with thymoma or thymic carcinoma are aged $40-60$ years $(2,3)$. The World Health Organisation (WHO) classification is based on histological analysis of epithelial cell atypia and the degree of infiltration of non-neoplastic lymphocytes (4). Although the great majority of these tumors exhibit more conventional histological characteristics, unusual types have also been described, such as sclerosing thymoma (5). Spontaneous regression (SR) of thymoma without therapy has been reported, although its incidence is rare (6-8). This is the case report of a patient with thymoma exhibiting SR and disappearance of the pleural effusion.

\section{Case report}

A 30-year-old man was admitted to Toho University Omori Medical Center with right chest pain and low-grade fever. The patient was a never-smoker and had no medical history. On physical examination, the temperature was $37.8^{\circ} \mathrm{C}$ and the respiratory sounds were clear. A chest X-ray revealed a mass shadow in the right hilum and a blunted right costophrenic angle. A chest X-ray obtained 3 months prior had shown minor expansion of the right mediastinum, without any findings of right pleural effusion, but no additional examinations were performed. A chest computed tomography (CT) scan revealed an anterior mediastinal tumor sized $11.0 \times 6.0 \times 5.0 \mathrm{~cm}$, with a right pleural effusion (Fig. 1). The laboratory analysis findings were normal, except for elevations of the white blood cell count $(11,000 / \mu 1)$, C-reactive protein $(4.1 \mathrm{mg} / \mathrm{dl})$ and serum cytokeratin fragment (CYFRA; $12.7 \mathrm{ng} / \mathrm{ml}$, normal, <2 ng/ml). Other tumor markers, including carcinoembryonic antigen, $\alpha$-fetoprotein, soluble interleukin- 2 receptor, human chorionic 
gonadotropin- $\beta$, and the anti-acetylcholine receptor antibody, were within normal limits.

On the day of admission, thoracocentesis was performed and $30 \mathrm{ml}$ of yellowish, slightly bloody effusion were obtained. The level of CYFRA in the pleural effusion was increased to $143 \mathrm{ng} / \mathrm{ml}$, the bacterial culture was negative and the cytological examination revealed no malignant cells. The patient exhibited no myasthenic symptoms, such as ptosis or muscle weakness. On the first day after admission (6 days after the initial CT), CT-guided needle biopsy (CTNB) was performed. The chest CT prior to performing a CTNB revealed a mild regression of the tumor $(10.0 \times 5.5 \times 4.4 \mathrm{~cm}$; reduction rate, $26.7 \%)$ with a marked decrease of the pleural effusion volume, without additional drainage (Fig. 2). The biopsied specimen was mostly necrotic and the pathological diagnosis was not definitive. Seven days later (13 days after the initial CT), further regression was evident on CT $(9.5 \times 5.4 \times 4.2 \mathrm{~cm}$; reduction rate, $34.7 \%)$ with disappearance of the pleural effusion.

At 35 days after the initial CT, the tumor had shrunk to $8.0 \times 3.6 \times 3.0 \mathrm{~cm}$ (reduction rate, $73.8 \%$ ) and the pleural effusion had completely disappeared without treatment (Fig. 3). The serum CYFRA level had decreased to $0.8 \mathrm{ng} / \mathrm{ml}$. A non-steroidal drug was administered for the right chest pain and fever. The patient's symptoms gradually disappeared and the laboratory markers of inflammation decreased. Although the mediastinal mass had shrunk without treatment, it did not completely disappear (Table I). The possibility of malignancy could not be excluded and the patient underwent surgical resection 62 days after the initial $\mathrm{CT}$. Magnetic resonance imaging, 2 days prior to surgery, revealed a high-intensity area in the left portion of the tumor, in fat-suppressed T1- and T2-weighted images, suggesting a cystic structure filled with considerable protein and serum (Fig. 4). The remaining portion of the tumor exhibited a solid pattern, with a mildly hyperintense signal in diffusion images and T2-weighted images. The tumor boundary was relatively sharp, without any definitive findings suggesting invasion.

The mediastinal mass adhered densely to the upper lobe of the right lung and thymectomy and partial resection of the right upper lobe were performed. The mediastinal mass was composed of a solid part and cystic space (Fig. 5A). In the solid part, there was epithelial cell proliferation admixed with a large number of lymphocytes, which was compatible with WHO type B2 thymoma (Fig. 5B). The tumor displayed coagulation necrosis and fibrosis, with foamy cell aggregations and cholesterol crystals (Fig. 5C). Microscopic transcapsular invasion was present (Masaoka classification, stage II). By contrast, the cystic wall was lined by a flattened epithelium including a small number of goblet cells. Thymic tissue with Hassall's corpuscles was attached to the thin-walled cyst, which was consistent with a thymic cyst. On immunohistochemical staining (Fig. 5D and E), the tumor was strongly positive for cytokeratin. The postoperative course was uneventful and the patient remains recurrence-free at 1 year after surgery.

\section{Discussion}

SR has been reported in various types of cancer, although it is considered unusual $(9)$. Cole $(9,10)$ reported that immunological reactions play an important role in this rare

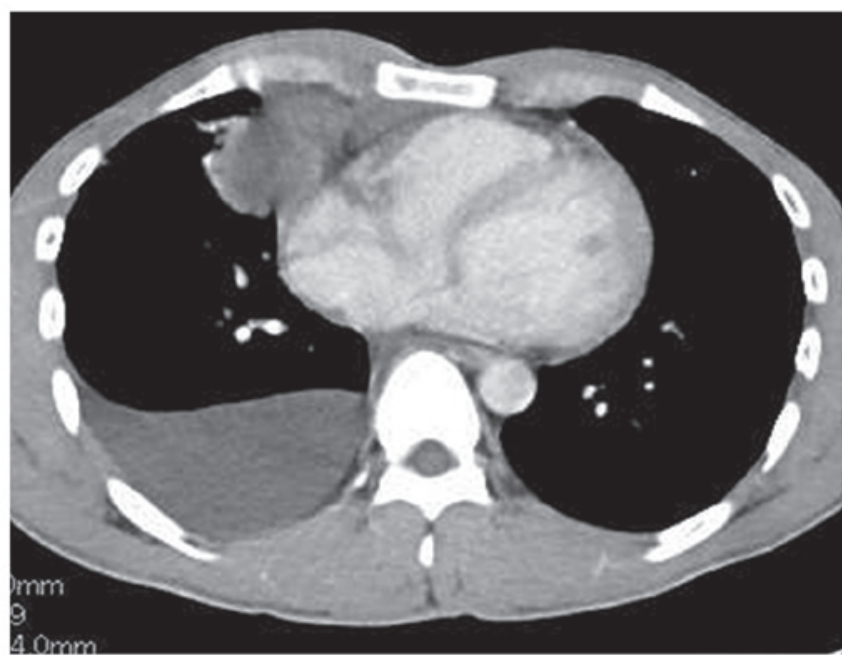

Figure 1. Chest computed tomography on admission, showing a large anterior mediastinal tumor $(11.0 \times 6.0 \times 5.0 \mathrm{~cm})$ with a right pleural effusion.

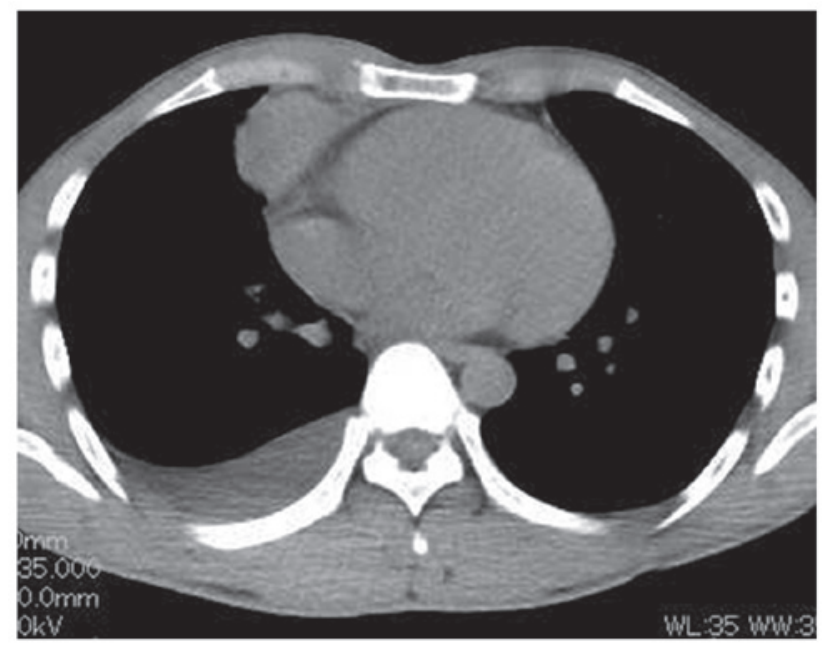

Figure 2. Chest computed tomography (CT) prior to CT-guided needle biopsy, showing spontaneous regression (reduction rate, 26.7\%) of the tumor, with marked decrease of the right pleural effusion volume.

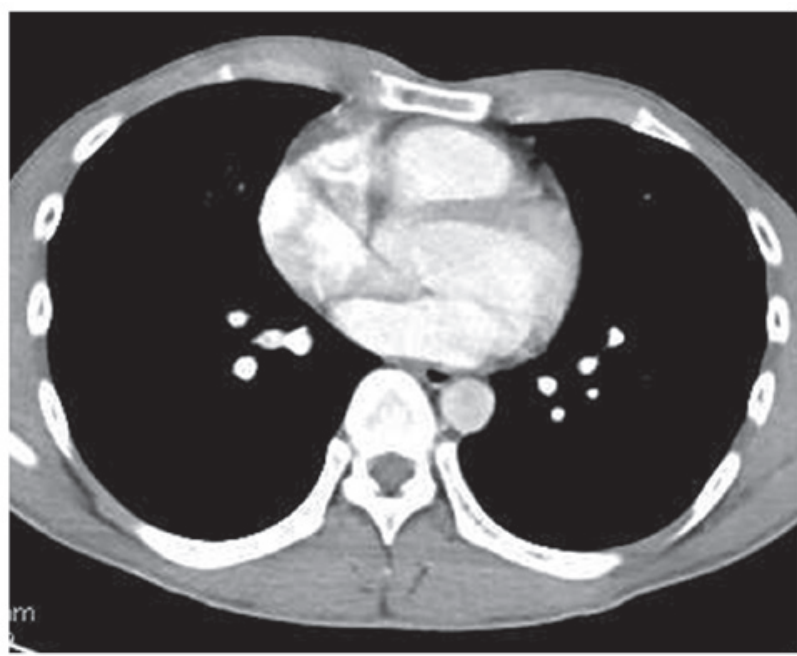

Figure 3. Chest computed tomography prior to surgery revealed marked regression (reduction rate, $73.8 \%$ ) of the tumor, with disappearance of the right pleural effusion. 
Table I. Serial changes in the serum levels of CYFRA and the size of the thymoma and volume of the pleural effusion on chest CT.

\begin{tabular}{|c|c|c|c|c|c|}
\hline Time points & Day & Size, mm & Reduction rate, $\%$ & Pleural effusion & CYFRA, ng/ml \\
\hline At first visit & 0 & $110 \times 60 \times 50$ & - & + & 12.7 \\
\hline At CTNB & 6 & $100 \times 55 \times 44$ & 26.7 & + & - \\
\hline After CTNB (7 days) & 13 & $95 \times 54 \times 42$ & 34.7 & - & - \\
\hline After CTNB (1 month) & 35 & $80 \times 36 \times 30$ & 73.8 & - & 0.8 \\
\hline
\end{tabular}

CT, computed tomography; CYFRA, cytokeratin fragment; CTNB, CT-guided needle biopsy.
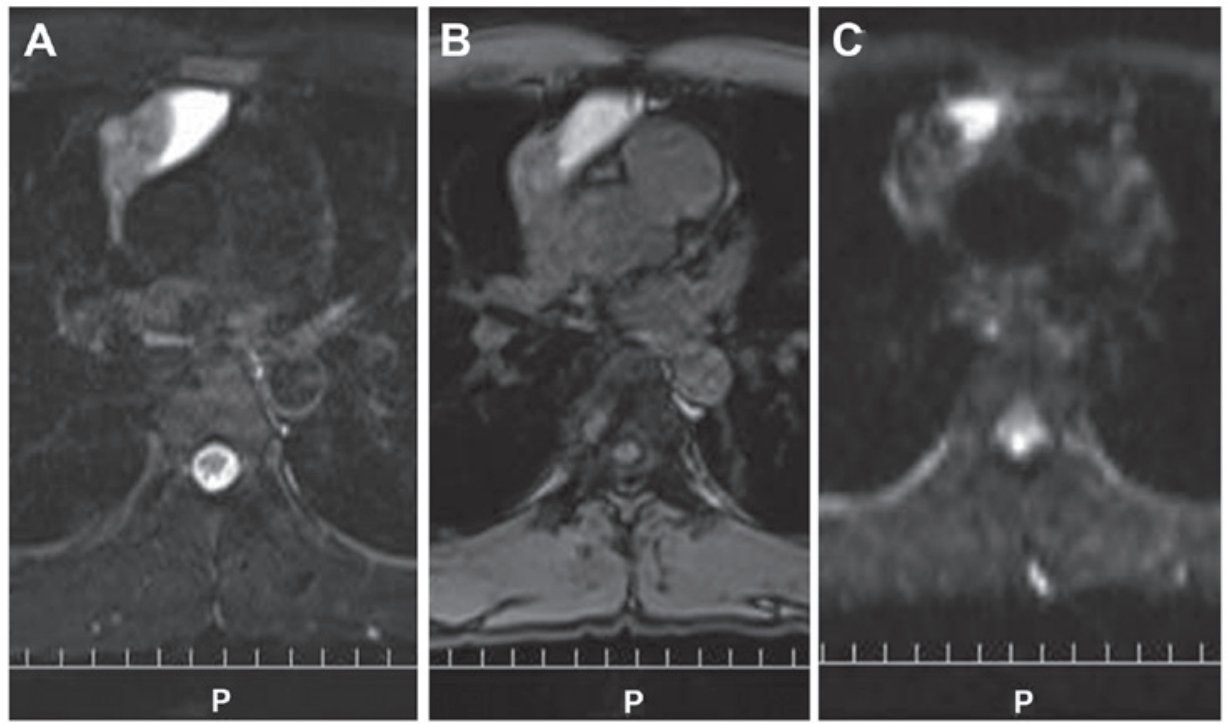

Figure 4. The left side of the tumor shows a high signal on (A) T2-weighted imaging and (B) fat suppression T1-weighted imaging, suggesting high protein and serum content of the cystic structure. The remaining portion of the tumor shows a mild high signal on (C) diffusion weighted images and T2-weighted images, suggesting a solid component.
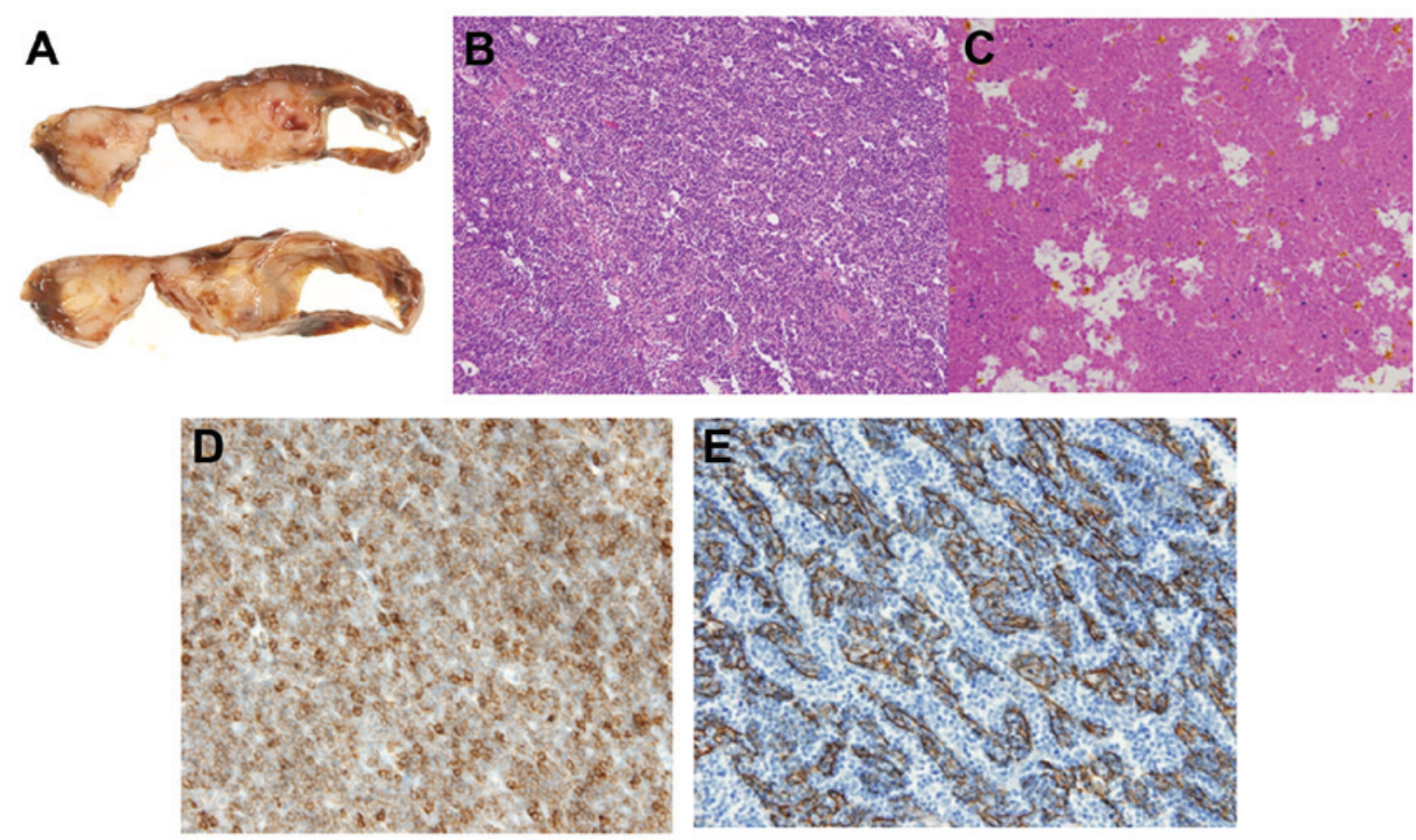

Figure 5. (A) Macroscopically, the resected specimen was composed of a solid mass with massive necrosis and a cystic space; (B) the mass in the right lobe is composed of epithelial cells admixed with lymphocytes; (C) the tumor included prominent degenerative and necrotic areas; (D) on CD5 immunohistochemical staining, the presence of $\mathrm{T}$ lymphocytes was demonstrated; (E) immunohistochemical staining was strongly positive for cytokeratin (AE1/AE3). 
event (10) and SR of tumors may be caused by necrosis and rupture (9). SR in mediastinal tumors has been reported in renal cell carcinoma, malignant melanoma and neuroblastoma (9). However, SR of thymoma is rare (6-8). Moran and Suster (1) reported 25 cases of thymoma with prominent hemorrhagic and necrotic changes, without a detailed description of tumor regression.

Thymoma is generally asymptomatic (1) and the majority of the patients with thymoma commonly present with an abnormal chest shadow, while $15 \%$ of the cases with thymoma are associated with myasthenia gravis (11). By contrast, all the reported patients with SR of thymoma have presented with symptoms, such as fever or chest pain (Table II). These symptoms may be associated with rapid tumor enlargement prior to regression, as was the case in our patient. One possible cause of the rapid enlargement of thymoma prior to regression has been reported to be disruption of the vascular supply and necrosis and an inflammatory reaction resulting in pleural effusion (6). All the patients with SR of thymoma presented with pleural effusion (Table II), supporting this hypothesis. In the present case, the tumor included prominent degenerative and necrotic areas, which was consistent with previous reports (Table II). Kuo (12) reported that a hemorrhagic and necrotic thymoma may develop into a sclerosing thymoma, due to the absorption of the hemorrhagic and necrotic components. Clinical findings such as fever, chest pain and elevation of serum levels of inflammatory markers are consistent with massive necrosis (12). The cause, mechanism and trigger of regression, as well as the cause of necrosis, have not been clearly determined and remain a hypothesis. Rapid tumor growth may lead to increased internal pressure, resulting in massive necrosis and subsequent tumor regression. In some patients, non-steroidal anti-inflammatory drugs were administered for subjective symptoms. Thymoma regression during corticosteroid treatment has been reported (4) and may be associated with stress and increased steroid levels. Some cases are believed to be caused by vascular insufficiency and thrombus formation (8), which was not observed in our patient.

Elevation of CYFRA in the pleural effusion was prominent in the present case, suggesting that the origin of CYFRA may be the epithelial cells of the thymoma. CYFRA has been reported to be a sensitive and specific marker for squamous cell lung cancer, through detecting a fragment of the cytokeratin-19, which is a subunit of the cytokeratin intermediate filament expressed in simple epithelia and their malignant counterparts (13). In the present case, the level of CYFRA was increased in the serum and pleural effusion, which had not been previously reported (Table II). The increased CYFRA levels may be attributed by a collapse of tumor cells and increased cytokeratin fragment modification by intracellular activated protease (14).

Cytokeratin is one of the intermediate filament proteins from the cytoskeleton of epithelial cells (15) and the results of cytokeratin staining in this case also suggests that elevation of CYFRA is caused by the thymoma. Originally, CYFRA was used in the diagnosis, assessment of therapeutic effectiveness and evaluation of prognosis in cancers such as squamous cell carcinoma (16). Additionally, the present case demonstrated that CYFRA may be useful for the evaluation of thymoma with extensive necrosis. The serum CYFRA levels decreased 
without treatment in our patient, possibly reflecting the decreased tumor volume or massive necrotic reaction.

We herein report a case of SR of thymoma. Although the frequency of this event is low, SR does not exclude malignancy and surgical intervention should be considered for the residual lesion.

\section{References}

1. Moran MD and Suster S: Thymoma with prominent cystic and hemorrhagic changes and areas of necrosis and infarction: A clinicopathologic study of 25 cases. Am J Surg Pathol 25: 1086-1090, 2001.

2. Engels EA and Pfeiffer RM: Malignant thymoma in the United States: Demographic patterns in incidence and associations with subsequent malignancies. Int J Cancer 105: 546-551, 2003.

3. Schmidt-Wolf IG, Rockstroh JK, Schüller H, et al: Malignant thymoma: Current status of classification and multimodality treatment. Ann Hematol 82: 69-76, 2003.

4. Barratt S, Puthucheary ZA and Plummeridge M: Complete regression of a thymoma to glucocorticoids, commenced for palliation of symptoms. Eur J Cardiothorac Surg 31: 1142-1143, 2007.

5. Moran CA and Suster S: 'Ancient' (sclerosing) thymomas: A clinicopathologic study of 10 cases. Am J Clin Pathol 121: 867-871, 2004.

6. Yutaka Y, Omasa M, Shikuma K, Okuda M and Taki T: Spontaneous regression of an invasive thymoma. Gen Thorac Cardiovasc Surg 57: 272-274, 2009.
7. Okagawa T, Uchida T and Suyama M: Thymoma with spontaneous regression and disappearance of pleural effusion. Gen Thorac Cardiovasc Surg 55: 515-517, 2007.

8. Fukui T, Taniguchi T, Kawaguchi K and Yokoi K: Spontaneous regression of thymic epithelial tumours. Interact Cardiovasc Thorac Surg 18: 399-401, 2014.

9. Cole WH: Spontaneous regression of cancer: The metabolic triumph of the host? Ann NY Acad Sci 230: 111-141, 1974.

10. Cole WH: Efforts to explain spontaneous regression of cancer. J Surg Oncol 17: 201-209, 1981.

11. Romi F: Thymoma in myasthenia gravis: From diagnosis to treatment. Autoimmune Dis 2011: 474512, 2011.

12. Kuo T: Sclerosing thymoma - a possible phenomenon of regression. Histopathology 25: 289-291, 1994.

13. Pujol JL, Grenier J, Daurès JP, Daver A, Pujol H and Michel FB: Serum fragment of cytokeratin subunit 19 measured by CYFRA 21-1 immunoradiometric assay as a marker of lung cancer. Cancer Res 53: 61-66, 1993.

14. Morita T, Kikuchi T, Hashimoto S, Kobayashi Y and Tokue A: Cytokeratin-19 fragment (CYFRA 21-1) in bladder cancer. Eur Urol 32: 237-244, 1997.

15. Hsu JD, Yao CC, Lee MY, et al: True cytokeratin 8/18 immunohistochemistry is of no use in distinguishing between primary endocervical and endometrial adenocarcinomas in a tissue microarray study. Int J Gynecol Pathol 29: 282-289, 2010.

16. Rastel D, Ramaioli A, Cornillie F and Thirion B: CYFRA 21-1, a sensitive and specific new tumour marker for squamous cell lung cancer. Report of the first European multicentre evaluation. CYFRA 21-1 Multicentre Study Group. Eur J Cancer 30A: 601-606, 1994. 